\section{Heavenly homes}

\author{
The discovery of our Galaxy's place in the
} Universe adds detail to our address.

A s Tim Radford, former science editor of The Guardian newspaper, noted in his 2011 book The Address Book, inquisitive schoolchildren the world over have a certain ritual. Handed a new exercise book and asked to add their name and address, they do so on a Universal scale. House number, street, town and country, postcode even, are followed by their designated continent and the name of our planet. Up the cosmic scale they go, noting the Solar System then the Milky Way, before offering the final identifier: the Universe.

It sounds precise; it is anything but. Most difficult for a deep-space postal service would be the first jump, from the infinite stretch of the known Universe down to our local Galaxy, the Milky Way. Well, things just got a little easier. (Although perhaps not for Radford, who may need to update his book.) This week, scientists add a new line to our planetary coordinates: the Laniakea galaxy supercluster.

Do not bother googling the name. It really is brand new, coined by an international group of astronomers on page 71 of this issue. Our place in the Universe, for so long one of the core mysteries of human existence that scientists and this journal are dedicated to unravelling, just got a little clearer. Laniakea, the scientists write, is our home supercluster, the one in which the Milky Way resides.

What kind of home is it? It is big - some 160 million parsecs across. Although not as big as some superclusters, it is the largest in our local neighbourhood, which is surprisingly crowded given the vast emptiness of most of the cosmological void. Laniakea has

several supercluster neighbours, including Coma, Perseus-Pisces and Shapley. (Together they make what? A super-supercluster? A hypercluster?)

It is a home that has been hiding in plain sight, colossal and all around us, yet unnoticed by previous astronomical surveys. As Elmo Tempel discusses in a News \& Views article on page 41, this is probably because the boundaries of superclusters are tricky to pin

"It is a home that has been hiding in plain sight, colossal and all around us, yet unnoticed by previous astronomical surveys." down, even for astronomers.

Laniakea was finally spotted with the help of what Tempel calls a "nifty algorithm" that helped the astronomers to turn incomplete measurements of the motion of galaxies into a map of the distribution and dynamics of cosmic matter. Their map shows galaxy superclusters as hotspots basins of attractions in fields of velocity flow - that can be hived off from their surroundings.

It is a local map. The nifty algorithm is limited because it depends on direct measurements from Earth of how rapidly galaxies recede owing to cosmic expansion. The rest of the Universe - those galaxies far, far away - remains uncharted territory, for now.

Still, the Laniakea survey is more than cartographical and geographical information. It reveals details of the large-scale structures that surround the Milky Way (best viewed in a video available here: go.nature.com/hpjzwh), which should help astronomers to close in their determinations of cosmological parameters such as the density of dark energy, the hidden power believed to push the Universe away from us.

The name Laniakea has Hawaiian roots, and roughly translated means spacious heaven. It is a beautiful address to have. And one that comes just in time for the new school year and a new curious generation..

\section{The digital toolbox}

\section{A new section of Nature examines the software and websites that make research easier.}

A sked to list the essential tools of a scientist's trade, most would probably think first of hardware: the microscope, the telescope, the mass spectrometer, the genome sequencer, the test-tube. But just as important to today's data-wranglers are software - Excel, ChemDraw, MATLAB - and the programming languages used to create it, such as Python, R and SQL. Such tools are integral to modern research practice, whether for analysing or visualizing data, sharing files, collaborating, writing up papers, publishing, searching the literature or simply organizing one's work. And although software engineers have often overlooked science in favour of more lucrative markets - think Flappy Bird, Instagram and iTunes - software, websites and apps designed specifically for researchers are blossoming.

Partly in response to this flourishing sector, Nature this week introduces a new section to help readers keep up to date. The Toolbox pages will collect the journal's writing on software tools and websites that researchers use to work more efficiently, or in new ways. Find them online at nature.com/toolbox, and monthly in print.

Barely a week goes by without the appearance of a website offering improved research productivity, or the launch of a start-up firm hoping that its unique idea will change scientific workflow. Toolbox will aim to guide the perplexed through the maze of sites and programs, discussing their similarities and distinguishing points. But it will be a community-driven resource, with scientists in various fields who work heavily with data or programs offering thoughts on their most commonly used software. The site will also collect Nature's writing on the broader context of online research - from open data to citizen science and crowd-funding.

In this issue, for instance, the section reviews a recent trend: the emergence of 'recommendation engines' that sift the flood of literature so that scientists can find relevant papers and information (see page 129). Future articles will include a look at the iPython interactivecomputing project and its applications for scientists, and an examination of websites that promise to help researchers to collaborate on research papers.

A little software literacy can make any researcher's daily life more efficient. Version-control systems such as Git, for instance, help to record changes made to files, to allow recall and analysis of past and evolving work. Websites such as GitHub (a favourite of software engineers, but increasingly of scientists too) build on these platforms to help researchers to work collaboratively on a research paper, or to ensure that data analysis is clear and reproducible.

Such programming tools are already the daily bread of the data scientists, bioinformaticians and climate modellers among Nature's readers. But jargon can make them off-putting for non-coders. At the same time, it can be hard to tell which of the more polished, graphically friendly software packages are worth investing your time in. So on the Toolbox website, scientists will be able to share their recommendations for particular software (both commercial and free). As a $\rightarrow$ NATURE.COM To comment online, click on Editorials at: go.nature.com/xhunqv taster, this week the Software Carpentry movement, which teaches basic software skills to researchers, explains its motivations and operations. A bad workman may blame his tools; a good scientist needs to keep track of them. 